Yu.M. Vasetsky

\title{
PENETRATION OF NON-UNIFORM ELECTROMAGNETIC FIELD INTO CONDUCTING BODY
}

The study is based on the exact analytical solution for the general conjugation problem of three-dimensional quasi-stationary field at a flat interface between dielectric and conducting media. It is determined that non-uniform electromagnetic field always decreases in depth faster than uniform field. The theoretical conclusion is confirmed by comparing the results of analytical and numerical calculations. The concept of strong skin effect is extended to the case when penetration depth is small not only compare to the characteristic body size, but also when the ratio of the penetration depth to the distance from the surface of body to the sources of the external field is small parameter. For strong skin effect in its extended interpretation, the influence of external field non-uniformity to electromagnetic field formation both at the interface between dielectric and conducting media and to the law of decrease field in conducting half-space is analyzed. It is shown, at the interface the expressions for the electric and magnetic intensities in the form of asymptotic series in addition to local field values of external sources contain their derivatives with respect to the coordinate perpendicular to the interface. The found expressions made it possible to generalize the approximate Leontovich impedance boundary condition for diffusion of non-uniform field into conducting half-space. The difference between the penetration law for the non-uniform field and the uniform one takes place in the terms of the asymptotic series proportional to the small parameter to the second power and to the second derivative with respect to the vertical coordinate from the external magnetic field intensity at the interface. References 25, figures 8 .

Key words: three-dimensional electromagnetic field, electromagnetic field formation, exact analytical solution, skin effect.

Дослідження засноване на точному аналітичному розв'язку загальної задачі спряження тривимірного квазістаціонарного поля на межі розділу діелектричного і електропровідного середовищ. Встановлено, що неоднорідне електромагнітне поле завжди зменшується в глибині швидще, ніж однорідне. Теоретичний висновок підтверджується зіставленням результатів аналітичних і чисельних розрахунків. Поняття сильного скін-ефекту поширюється на випадок, коли глибина проникнення мала порівняно не тільки з характерними розмірами тіла, але також коли відношення глибини проникнення до відстані від поверхні тіла до джерел зовнішнього поля є малим параметром. Для сильного скін-ефекту в його розширеної інтерпретації проаналізовано вплив неоднорідності зовнішнього поля на формування електромагнітного поля на межі $і$ на закон зменшення поля в провідному півпросторі. Показано, щзо на межі вирази у вигляді асимптотичних рядів крім локальних значень поля зовнішніх джерел містять їх похідні по координаті, перпендикулярної граничної поверхні. Отримані вирази дозволили узагальнити наближену імпедансну граничну умову Леонтовича для дифузї̈ неоднорідного поля в провідний півпростір. Відмінність законів проникнення для неоднорідного і однорідного полів має місие в членах асимптотичного ряду, пропориійних малому параметру в другому ступені і другій похідній по вертикальній координаті від напруженості зовнішнього магнітного поля у граничній поверхні. Бібл. 25 , рис. 8.

Ключові слова: тривимірне електромагнітне поле, формування електромагнітного поля, точний розв'язок задачі, скін-ефект.

Исследование основано на точном аналитическом решении общей задачи сопряжения трехмерного квазистационарного поля на границе раздела диэлектрической и проводящей сред. Установлено, что неоднородное электромагнитное поле всегда убывает по глубине быстрее, чем однородное. Теоретический вывод подтверждается сопоставлением результатов аналитических и численных расчетов. Понятие сильного скин-эффекта распространяется на случай, когда глубина проникновения мала по сравнению не только с характерными размерами тела, но также когда отношение глубины проникновения к расстоянию от поверхности тела до источников внешнего поля является мальм параметром. Для сильного скинэффекта в его расширенной интерпретачии проанализировано влияние неоднородности внешнего поля на формирование электромагнитного поля на границе и на закон убывания поля в проводящем полупространстве. Показано, что на границе выражения в виде асимптотических рядов для напряженностей полей помимо локальных значений поля внешних источников содержат их производные по координате, перпендикулярной граничной поверхности. Полученные выражения позволили обобщить приближенное импедансное граничное условие Леонтовича для диффузии неоднородного поля в проводящее полупространство. Отличие законов проникновения для неоднородного и однородного полей имеет место в членах асимптотического ряда, пропорциональных малому параметру во второй степени и второй производной по вертикальной координате от напряженности внешнего магнитного поля у граничной поверхности. Библ. 25 , рис. 8.

Ключевые слова: трехмерное электромагнитное поле, формирование электромагнитного поля, точное решение задачи, скин-эффект.

Introduction. The interaction of electromagnetic field with conducting medium is the subject of study in many technical and electrophysical applications. Examples include equipment for high frequency induction heat treatment of metals [1-3], installations for processing of metals under the action of high intensity electromagnetic field and high density currents [4-6], devices for electromagnetic forming or high-speed forming technology using pulse magnetic field [7-9]. A strong skin effect occurs in conducting elements of this

equipment, in which the current and electromagnetic field are concentrated in a thin skin layer. The features of the electromagnetic field penetration into a conducting body, including its decrease in depth, depend not only on the electrical conductivity, the relative magnetic permeability of the medium and the field frequency, but also on the geometric properties of boundary surfaces and the character of the field distribution at the surface. Also, in the mentioned devices the wavelength of the (c) Yu.M. Vasetsky 
electromagnetic field is usually much larger than any characteristic dimensions of the system and the processes can be considered quasi-stationary, in which wave phenomena can be neglected.

These two circumstances determine the main limitations - it is considered the formation of a quasistationary electromagnetic field in systems with strong skin effect. Under the indicated limitations as it note in [10], the use of simplified approaches to the calculation of specific problems, and their use in the development of a number of numerical methods are of methodological and practical importance. Despite the long history of development, the study of the formation of electromagnetic field with strong skin effect remains an actual task.

Approximate calculation methods are often used to determine the electromagnetic field with strong skin effect. For body of infinite conductivity the penetration depth is equal to zero $\delta \rightarrow 0$, and it is sufficient to use a mathematical model in which the tangential component of the electric field intensity and the normal component of the magnetic field intensity are equal to zero at the surface of the conducting body [11, 12]. The finite penetration depth is taken into account in approximate mathematical models using the concept of the impedance boundary condition formulated by $\mathrm{M}$. Leontovich [13]. It is assumed that locally the electromagnetic field penetrates into a metal body in the same way as a uniform field penetrates into a conducting half-space. The local values of the electromagnetic field at the interface correspond to the model body with perfect conductivity.

Based on the perturbation method, it became possible to calculate the fields inside and outside of conductors with a curved surface [14]. Using integral equations for curved surfaces, the solution of the problem in the second-order approximation was obtained in [15]. The expansion in a power series in a small parameter proportional to the depth of field penetration includes the Leontovich condition as a first-order approximation. The field penetration depth for such conductors dependents on the average surface curvature [16]. For curved conductors, first- and second-order corrections to the field distribution corresponding to the diffusion of uniform field into conducting half-space are found in [17].

The concept of surface impedance makes it possible to use it in modeling problems of electrodynamics, taking into account the geometric and physical properties of real boundary surfaces. Detailed results of research in this direction are given in a number of reviews. For example, in [18] the experience of many years of research on the application of the impedance approach in mathematical modeling is systematized. The article describes the types of structures for which methods of theoretical determination of the values of surface impedances are known. The generalized boundary conditions for the analytical determination of the electromagnetic field characteristics at the interface between media with twodimensional inhomogeneities are analyzed in [19]. A comprehensive analysis of studies of the skin effect in problems of electrodynamics is presented in the book [10] where, among other things, the systematic method for constructing boundary conditions of any order based on a perturbation approach is considered, general approaches to the numerical methods application are formulated such as the boundary integral equations method, the finite element method, and the finite difference method, and also specific examples of calculations are presented.

In most of the cited papers, mathematical models of the diffusion of non-uniform electromagnetic field are limited of a small penetration depth value or insignificant field non-uniformity at the body surface. The exact solution of the problem of the diffusion of non-uniform field into conducting half-space is presented in [17] for the specific case of a field created by a thin rectilinear conductor with a current directed parallel to the interface between the media. The exact solution made it possible to justify the limitations under which the impedance boundary condition is valid for the considered nonuniform field.

In [20], we obtained a complete analytical solution to the problem of the penetration of a three-dimensional quasi-stationary electromagnetic field created by external sources in the form of current contours of arbitrary configuration located near conducting half-space. There are no restrictions on field non-uniformity in the obtained solution. For the same mathematical model, an analytical solution is found for the electromagnetic field also in the dielectric half-space [21]. The exact solution made it possible to obtain some justified results of the electromagnetic field formation. In particular, the main property is that in a conducting half-space the current density and electric field intensity do not contain components perpendicular to the boundary surface for any system of initial currents and arbitrary dependence of currents on time. In addition, in a short report, it is noted as a general property that a non-uniform electromagnetic field is decreased in a conducting medium faster than a uniform field [22]. Analytical expressions are also obtained for the field intensities at the interface between the media, consequence of which is the generalization of the Leontovich approximate impedance boundary condition to the case of penetration of a non-uniform electromagnetic field into conducting medium [23]. The cited works contain separate parts of the problem of nonuniform field penetration into a conducting medium and do not sufficiently represent the solution of the problem for an arbitrary three-dimensional quasi-stationary electromagnetic field and for any properties of the media.

The purpose of this work is to generalize the results of studying the penetration of a three-dimensional nonuniform electromagnetic field into conducting half-space, which unlike many well-known studies, is based on exact analytical solution of the problem for an external field created by sources in the form of an arbitrary system of contours with alternating currents without restrictions on the properties of the media and the field frequency. The following objectives are to achieve the aim: substantiation of the consequence that a non-uniform electromagnetic field decreases in depth always faster than an uniform field; investigation of the distribution of a non-uniform electromagnetic field at the interface between dielectric and conductive media; estimation of the influence of field non-uniformity on its distribution in the skin layer in the case of strong skin effect. 
Mathematical model and analytical solution of the three-dimensional problem. The present work differs from most of the previous studies in that it is based on a complete analytical solution of the three-dimensional problem of the electromagnetic field in an enough general formulation $[20,21]$ and this allows to obtain a number of substantiated general consequences. Note that numerical methods, which also make it possible not to limit the penetration depth, presuppose a specific formulation of problems, and conclusions usually do not go beyond the performed calculations.

Mathematical model for a single contour with current. The analytical solution is obtained for the linear problem of conjugation at a plane interface between dielectric and conducting media of the three-dimensional quasi-stationary field. The solution satisfies Maxwell's equations and boundary conditions including the equality of the normal components of the conduction current density in a conducting medium and the displacement current density in a dielectric medium. It is based on the well-known analytical solution of the problem for an emitting current dipole near the interface. A closed contour $l$ located in a nonconducting nonmagnetic medium with a relative dielectric permittivity $\varepsilon_{e}$, without loss of generality, was represented by a serial system of dipoles with a constant initial current $\dot{I}_{0}$ along the contour. A conducting body is modeled as a half-space with electrical conductivity $\gamma$ and relative magnetic permeability $\mu$, in which eddy currents are induced.

The element of the external current contour is shown in Fig. 1 as a segment of curve in the upper half-space $z>0$. The position of the field source point on the contour $M$ relative to the observation point $Q$ is determined by vector $\boldsymbol{r}$. The axis $z$ is oriented perpendicular to the interface surface in the direction of the single vector $\boldsymbol{e}_{z}$. For an arbitrary spatial contour, the unit tangent vector to the contour $\boldsymbol{t}=\boldsymbol{t}_{\|}+\boldsymbol{t}_{\perp}$ has nonzero projections onto the vertical direction $\boldsymbol{t}_{\perp}=\left(\boldsymbol{t} \cdot \boldsymbol{e}_{z}\right) \boldsymbol{e}_{z}$ and onto the interface between the media $\boldsymbol{t}_{\|}=\boldsymbol{t}-\left(\boldsymbol{t} \cdot \boldsymbol{e}_{z}\right) \boldsymbol{e}_{z}$.

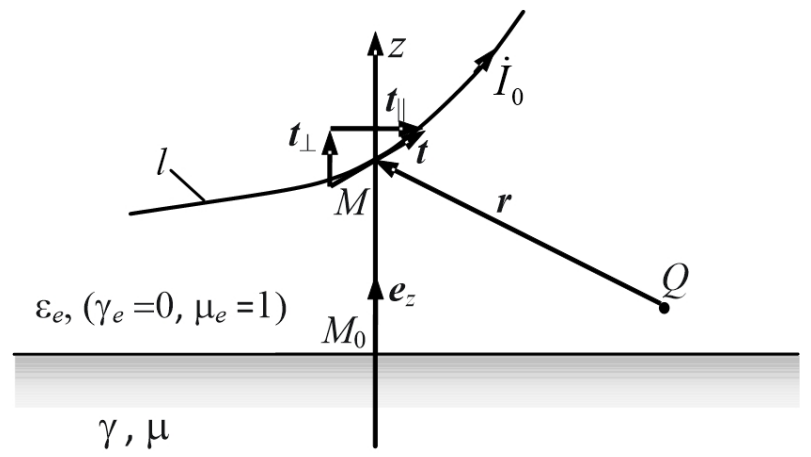

Fig. 1. Element of arbitrary spatial contour $l$ with current $\dot{I}_{0}$ located near conducting half-space

For the system under consideration all characteristics of the electromagnetic field in conducting and dielectric media are found in the form of expressions for the complex-value amplitudes of the vector and scalar potentials, the intensities of the electric and magnetic fields. (Complex-value amplitudes we will mark with a dot over the corresponding symbols). Since the linear problem is considered, it can easily be extended to the general case of an arbitrary external field created by the corresponding system of current contours and to an arbitrary dependence of currents on time $I_{0}(t)$ using the Fourier transform.

Electromagnetic field in conducting half-space. The expression for electric intensity in conducting half-space at point $Q(\rho, \theta, z)$ is the following [20]

$$
\dot{\boldsymbol{E}}_{i}=-\frac{\mu_{0} \dot{I}_{0}}{4 \pi} 2 i \omega \oint_{l}\left[\boldsymbol{t}_{\|} T_{1}(\rho, \theta, z)+\left(\boldsymbol{t} \cdot \boldsymbol{e}_{z}\right) \boldsymbol{e}_{\rho} T_{2}(\rho, \theta, z)\right] d l,
$$

where $\omega$ is cyclic frequency, $i$ is imaginary unit, $\mu_{0}$ is permeability of vacuum. Here the local cylindrical coordinates $(\rho, \theta, z)$ with its unit basis vectors $\left(\boldsymbol{e}_{\rho}, \boldsymbol{e}_{\theta}, \boldsymbol{e}_{z}\right)$ are used (Fig. 2). The center of the coordinate system is located at point $M_{0}$ intersection of the vertical axis with the interface. The angle $\theta$ is defined relative to the axis directed along the unit vector $\boldsymbol{e}_{\|}=\boldsymbol{t}_{\|} / \boldsymbol{t}_{\|} \mid$. The values of local coordinates depend on the position of the source point $M$ during integration along the contour. follows

The functions $T_{1}(\rho, \theta, z)$ and $T_{2}(\rho, \theta, z)$ in (1) are as

$$
\begin{aligned}
& T_{1}(\rho, \theta, z)=\int_{0}^{\infty} \exp (q z) \frac{\exp \left(-\vartheta z_{M}\right) J_{0}(\vartheta \rho)}{w(\vartheta)} \vartheta d \vartheta, \\
& T_{2}(\rho, \theta, z)=\int_{0}^{\infty} \exp (q z) \frac{\exp \left(-\vartheta z_{M}\right) J_{1}(\vartheta \rho)}{w(\vartheta)} \vartheta d \vartheta,
\end{aligned}
$$

where $q=\sqrt{\vartheta^{2}+p^{2}}, \quad p=\sqrt{i \omega \mu \mu_{0} \gamma}$ is propagation constant, $w(\vartheta)=\vartheta+q / \mu, J_{0}(\cdot)$ and $J_{1}(\cdot)$ are Bessel functions of the first kind of zero and first orders. Since the decrease of the field with respect to depth is considered, the functions that depend on the coordinate $z$ are distinguished by a separate factor in (2).

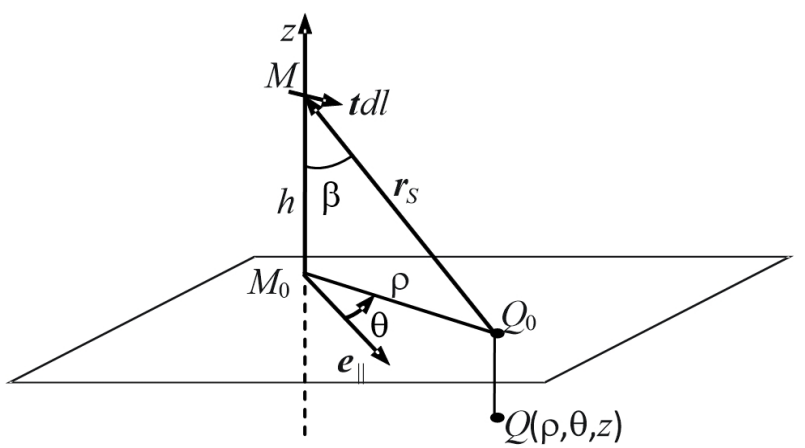

Fig. 2. Geometric parameters for determining the values of the electromagnetic field intensities at the point $Q(\rho, \theta, z)$ in conducting half-space $z<0$

The expression for the magnetic field intensity $\dot{\boldsymbol{H}}_{i}$ in conducting half-space follows from the Maxwell equation $\nabla \times \dot{\boldsymbol{E}}=-i \omega \mu \mu_{0} \dot{\boldsymbol{H}}$

$\dot{\boldsymbol{H}}_{i}=\frac{\mu_{0} \dot{I}_{0}}{4 \pi \mu} \oint_{l}\left[\left|\boldsymbol{t}_{\|}\right|\left[\boldsymbol{e}_{z} \sin \theta \frac{\partial T_{1}}{\partial \rho}+\left[\boldsymbol{e}_{\|} \times \boldsymbol{e}_{z}\right] \frac{\partial T_{1}}{\partial z}\right]-\left(\boldsymbol{t} \cdot \boldsymbol{e}_{z}\right) \boldsymbol{e}_{\theta} \frac{\partial T_{2}}{\partial z}\right] d l$.

As follows from (1), the projection of the electric field intensity to the direction perpendicular to the plane interface between the media is always equal to zero $\dot{\boldsymbol{E}}_{i} \cdot \boldsymbol{e}_{z}=0$. On the other hand, in this medium all 
components of the magnetic field intensity in the general case can have nonzero values.

To study other general features of the electromagnetic field formation associated with the penetration of nonuniform field into conducting half-space, it is advisable to introduce dimensionless parameters, whose values are due to the form of expressions (2). In this case, we use dimensionless integration variable $\chi=\vartheta \mu / \sqrt{\omega \mu \mu_{0} \gamma}$ and take into account that $p z=\sqrt{2 i} \frac{z}{\delta}$, where $\delta=1 / \operatorname{Re}(p)=\sqrt{2 /\left(\omega \mu \mu_{0} \gamma\right)}$ is the penetration depth of a uniform field into conducting half-space [24]. As a result, the functions $T_{1}(\rho, \theta, z)=\frac{p}{\mu} f_{1}\left(\frac{z}{\delta}, \varepsilon, \beta\right) \quad$ and $T_{2}(\rho, \theta, z)=\frac{p}{\mu} f_{2}\left(\frac{z}{\delta}, \varepsilon, \beta\right)$ will be expressed in terms of dimensionless parameters where

$$
\begin{aligned}
f_{1}\left(\frac{z}{\delta}, \varepsilon, \beta\right) & =\int_{0}^{\infty} K\left(\frac{z}{\delta}, \chi\right) \cdot \frac{\exp \left(-\frac{\chi \cos \beta}{\varepsilon}\right) J_{0}\left(\frac{\chi \sin \beta}{\varepsilon}\right)}{w_{1}(\chi)} \chi d \chi, \\
f_{2}\left(\frac{z}{\delta}, \varepsilon, \beta\right) & =\int_{0}^{\infty} K\left(\frac{z}{\delta}, \chi\right) \cdot \frac{\exp \left(-\frac{\chi \cos \beta}{\varepsilon}\right) J_{1}\left(\frac{\chi \sin \beta}{\varepsilon}\right)}{w_{1}(\chi)} \chi d \chi, \\
K\left(\frac{z}{\delta}, \chi\right) & =\exp (q z)=\exp \left(\sqrt{2 i} \frac{z}{\delta} \sqrt{1+\left(\frac{\chi}{\mu \sqrt{i}}\right)^{2}}\right),
\end{aligned}
$$

here the parameter $\varepsilon=\mu \delta / \sqrt{2} r_{S}$ is proportional to the ratio of the penetration depth $\delta$ to the distance $r_{S}$ from the field source at a point $M$ on the contour to the body surface at a point $Q_{0}$ (Fig 2). The denominator $w_{1}(\chi)$ in the integrands (4) is written as

$$
w_{1}(\chi)=\frac{\chi}{\sqrt{i}}+\sqrt{1+\left(\frac{\chi}{\mu \sqrt{i}}\right)^{2}} .
$$

Expressions (1) and (3) describe the penetration of the electromagnetic field of arbitrary contour with current into conducting half-space and in the general case they differ from approximate description of the penetration of uniform field. Both the values of the intensity of the electric and magnetic fields on the boundary surface $z=0$, and the law of their decrease depending on the coordinate $z$ are differed. Usually, in approximate models, the initial value is the tangential component of the magnetic field intensity $\dot{\boldsymbol{H}}_{\tau}$ at the boundary, the local value of which for a body of arbitrary shape is found from the solution of the external problem under the assumption of the perfect skin effect $\delta \rightarrow 0$ [11]. The local value on the body surface $\dot{\boldsymbol{H}}_{\tau}$ is taken as the value of the uniform field. Its penetration into conducting half-space is described by the known distribution of the electric $\dot{\boldsymbol{E}}_{i}$ and magnetic $\dot{\boldsymbol{H}}_{i}$ intensities: $\quad \dot{\boldsymbol{E}}_{i}=\dot{\boldsymbol{E}}_{\tau} e^{-p z}, \dot{\boldsymbol{H}}_{i}=\dot{\boldsymbol{H}}_{\tau} e^{-p z} \quad$ [24]. The field vectors are related by the Leontovich approximate impedance boundary condition $\dot{\boldsymbol{E}}_{\tau}=\varsigma\left[\boldsymbol{e}_{z} \times \dot{\boldsymbol{H}}_{\tau}\right]$, where the surface impedance $\varsigma=\sqrt{i \omega \mu \mu_{0} \gamma} / \gamma$ in this case connects the values of the field vectors not only at the interface between dielectric and conducting media $\dot{\boldsymbol{E}}_{\tau}, \dot{\boldsymbol{H}}_{\tau}$, but also in the entire conducting half-space $\dot{\boldsymbol{E}}_{i}, \dot{\boldsymbol{H}}_{i}$. In the general case of non-uniform electromagnetic field penetration, expressions (1) and (3) show the difference both from the values of the electric $\dot{\boldsymbol{E}}_{\tau}$ and magnetic $\dot{\boldsymbol{H}}_{\tau}$ intensities at the boundary surface with perfect skin effect, and the law of their decrease depending on the coordinate $z$.

Electromagnetic field in dielectric half-space. The expressions for electric $\dot{\boldsymbol{E}}_{e}$ and magnetic $\dot{\boldsymbol{H}}_{e}$ intensities in dielectric half-space where the current contour is located are determined by single function $G_{e}$ [21]

$$
\begin{aligned}
& \dot{\boldsymbol{E}}_{e}=\dot{\boldsymbol{E}}_{e 1}+\dot{\boldsymbol{E}}_{e 2}+\dot{\boldsymbol{E}}_{e 3}=-i \omega \frac{\mu_{0} \dot{I}_{0}}{4 \pi} \oint_{l}\left(\frac{\boldsymbol{t}}{r}-\frac{\boldsymbol{t}_{1}}{r_{1}}-\boldsymbol{e}_{z} \times\left[\boldsymbol{t}_{1} \times \nabla G_{e}\right]\right) d l, \\
& \left.\dot{\boldsymbol{H}}_{\boldsymbol{e}}=\dot{\boldsymbol{H}}_{\boldsymbol{e} 1}+\dot{\boldsymbol{H}}_{\boldsymbol{e} 2}+\dot{\boldsymbol{H}}_{\boldsymbol{e} 3}=-\frac{\dot{I}_{0}}{4 \pi} \oint \frac{\boldsymbol{t} \times \boldsymbol{r}}{r^{3}}-\frac{\boldsymbol{t}_{1} \times \boldsymbol{r}_{1}}{r_{1}^{3}}-\boldsymbol{t}_{1} \times \nabla\left(\frac{\partial G_{e}}{\partial z}\right)\right] d l,
\end{aligned}
$$

where function $G_{e}$ using dimensionless values are determined by following improper integral

$$
G_{e}=\frac{2}{\sqrt{i}} \int_{0}^{\infty} \frac{\exp \left(-\frac{\chi \cos \beta_{1}}{\varepsilon_{1}}\right) J_{0}\left(\frac{\chi \sin \beta_{1}}{\varepsilon_{1}}\right)}{w_{1}(\chi)} d \chi .
$$

The geometric quantities included in expressions (7) - (9) are shown in Fig. 3. The elements $t d l$ of the initial contour and $\boldsymbol{t}_{1} d l$ of the mirror reflection contour relative to the interface are located at the points $M$ and $M_{1}$ respectively. Projections of tangent vectors onto the vertical axis are equal in absolute value and opposite in their direction $\left(\boldsymbol{t}_{1 z}=-\boldsymbol{t}_{z}\right)$, and the projections $\boldsymbol{t}_{\|}$and $\boldsymbol{t}_{1 \|}$ onto the plane of interface between media are equal in their lengths and directions $\boldsymbol{t}_{1 \|}=\boldsymbol{t}_{\|}$, i.e. $\boldsymbol{t}=\boldsymbol{t}_{\|}+\boldsymbol{t}_{z}, \boldsymbol{t}_{1}=\boldsymbol{t}_{1 \|}+$ $+\boldsymbol{t}_{1 z}=\boldsymbol{t}_{\|}-\boldsymbol{t}_{z}$. Vectors $\boldsymbol{r}=\left(z_{M}-z\right) \boldsymbol{e}_{z}+\boldsymbol{\rho}$ and $\boldsymbol{r}_{1}=\left(z_{M 1}-z\right) \boldsymbol{e}_{z}+$ $+\boldsymbol{\rho}=-\left(z_{M}+z\right) \boldsymbol{e}_{z}+\boldsymbol{\rho}$ (the vector $\boldsymbol{\rho}$ is the projection of vector $\boldsymbol{r}$ or vector $\boldsymbol{r}_{1}$ onto interface) determine positions of points $M$ and $M_{1}$ relative to the observation point $Q$. The angle $\beta_{1}$ shows the orientation of the vector $\boldsymbol{r}_{1}$ relative to the vertical axis. The parameter $\varepsilon_{1}=\mu \delta /\left(\sqrt{2} r_{1}\right)$ is connected with distance $r_{1}$ between points $M_{1}$ and $Q$.

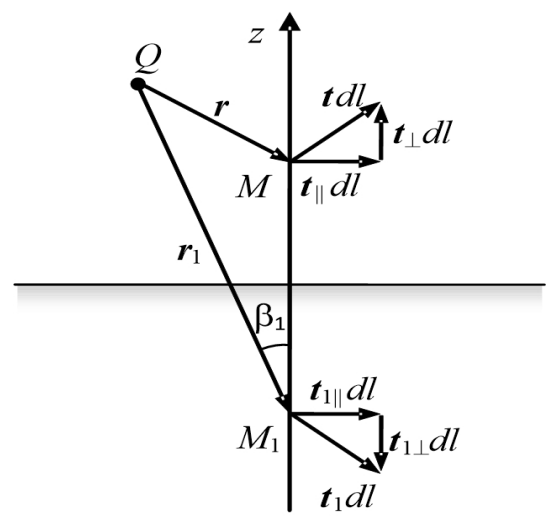

Fig. 3. Location the contour element $t d l$ and its mirror reflection $\boldsymbol{t}_{1} d l$ relative to the observation point $Q$ 
The first and second terms in (7) and (8) describe the solution of the problem for perfect skin-effect when $\delta \rightarrow 0$ [20]. At a value of the penetration depth other than zero the impact of electro-physical properties of the medium is taken into account by the third term.

The electromagnetic field (7) and (8) at an arbitrary point of the dielectric half-space is created by all sources, including the current of the initial contour, eddy currents in conducting medium, sources of magnetization of the medium and electric charges at the interface between the media. We can say that this total field decreases from the value at the interface during its diffusion into conducting body. The presence of the third term in (7) and (8) determines the difference between the tangential components of the field intensities on the surface from their values $\dot{\boldsymbol{E}}_{\tau}, \dot{\boldsymbol{H}}_{\tau}$, which correspond to the field in the approximate model of the perfect skin effect.

General feature of decrease of non-uniform electromagnetic field in the conducting half-space. The availability of the exact solution to the general problem allows, first of all, to analyze the features of the penetration of non-uniform field into a conducting halfspace without restrictions on the values of the electrophysical parameters and the field frequency.

As it follows from (2) and (4), the distribution of any component of the electric and magnetic field intensities in the skin-layer, depending on the coordinate $z$, is associated with exponential function $K(z / \delta, \chi)(5)$ in the integrands. The factor $\sqrt{1+[\chi /(\mu \sqrt{i})]^{2}}$ in the exponent affects to the field decrease law. If the influence of the second term $[\chi /(\mu \sqrt{i})]^{2}$ is absent, it corresponds to the decrease law of the uniform field. Since $\operatorname{Re}\left(\sqrt{1+[\chi /(\mu \sqrt{i})]^{2}}\right)>1$, the decrease of the nonuniform electromagnetic field created by the current contour is always faster than that of the uniform field. Taking into account the principle of superposition, this conclusion will be valid for any system of initial closed contours and therefore is valid in the general case of arbitrary external field.

Thus, faster decrease of non-uniform electromagnetic field as compared to uniform field is general feature of the electromagnetic field formation at its diffusion into conducting half-space. A qualitative explanation of the found feature can be based on the analysis of the inhomogeneous field formation under the action of «standard» external sources and it is the subject of additional research.

Let us consider the influence of the parameter $\varepsilon$ on the field penetration law, that is, the effect of the distance between the external field sources and the body surface in comparison with the penetration depth (at $\mu=1$ ). The parameter $\varepsilon$ also characterizes the field non-uniformity, since the closer the current contour is to the surface, the more non-uniform field is at its surface. This is reflected in the influence of the parameter $\varepsilon$ on the dependences of the functions $f_{1}(z / \delta, \varepsilon, \beta)$ and $f_{2}(z / \delta, \varepsilon, \beta)$ with respect to coordinate $z$ in (4).
Let, for example, the sources of the external field are remote at a considerable distance from the surface of a conducting body and, accordingly, for all points of the contour $\varepsilon<<1$. In this case, in (4), due to the presence of the exponential function $\exp (-\chi \cos \beta / \varepsilon)$, the value of the integrands turns out to be insignificant when $\chi \cos \beta>\varepsilon$. That is, the value of improper integrals (4) at small values $\varepsilon$ is mainly determined by the behavior of the integrand near the lower limit of integration $\chi=0$. This means that when integrating in (4), the influence of the factor $\sqrt{1+[\chi /(\mu \sqrt{i})]^{2}}$ will slightly differ from the case when this factor is equal to one. Therefore, if $\varepsilon<<1$, then the decrease in the field from its local value on the surface at the point $Q_{0}$ will be close to the decrease in the uniform field.

If the parameter $\varepsilon$ is not small the influence of the factor $\sqrt{1+[\chi /(\mu \sqrt{i})]^{2}}$ is much more. In this case, the elements of the contour as a source of the external field are located closer to the interface between the media and the decrease of the electromagnetic field will occur according to a different law with larger decrease rate in depth.

A specific example when the penetration depth $\delta=\sqrt{2 /\left(\omega \mu \mu_{0} \gamma\right)}$ is comparable to the dimensions of the contour illustrates the general conclusion of threedimensional field decrease. An additional argument for the validity of the conclusion can also be a comparison of the results of calculating the decrease of non-uniform electromagnetic field, performed using the obtained analytical expressions and using the numerical method in the Comsol package [22].

The calculation was performed for a circular contour located in a plane perpendicular to flat interface, as shown in Fig. 4. The radius of the contour is $R=0,05 \mathrm{~m}$, the minimum distance from the contour to the interface is $h_{0}=0,02 \mathrm{~m}$, the electrophysical properties of the medium are as follows: $\mu=1, \gamma=10^{5} 1 /(\Omega \cdot \mathrm{m})$. In contrast to the analytical method, in the numerical calculation, the current contour was selected in the form of a conductor with a square cross-section $2 r \times 2 r$ at $r=0,004 \mathrm{~m}$. In the numerical calculation, the problem was solved in a limited area, the dimensions of which significantly exceed the contour radius. Different values of the field penetration depth and, accordingly, the values of the ratio $\delta / R$ or $\varepsilon_{m}=\max (\varepsilon)=\mu \delta / \sqrt{2} h_{0}$ are obtained by choosing the field frequency.

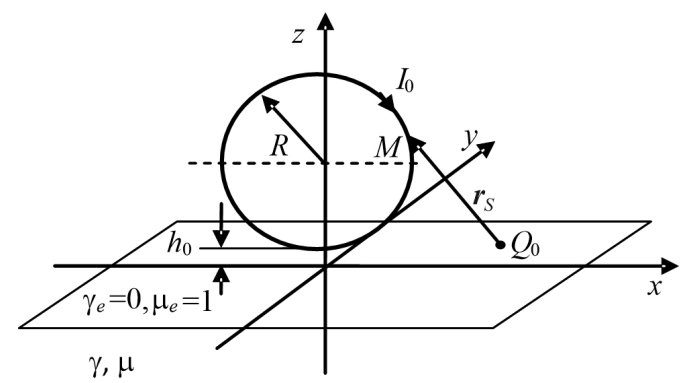

Fig. 4. Electromagnetic system with a circular current contour located in the plane perpendicular to the interface 
The results of calculating the electric and magnetic field intensities are shown in Fig. 5,a-c. In the upper group of figures, arrows show the distribution of the induced current density $\boldsymbol{j}_{i}=\gamma \boldsymbol{E}_{i}$ in the vertical plane passing through the center of the circular contour. The results of these calculations, performed by the numerical method, confirm the theoretical conclusion about the zero value of the vertical components of the electric field intensity and current density. The curves in the figures below show the change with depth for component of the amplitude of the electric field intensity, normalized to the value of the field at the surface $E_{x}^{*}=\left|\dot{E}_{x}\right| /\left|\dot{E}_{x}(z=0)\right|$. The coordinate value in conducting medium is normalized to the value of the field penetration depth. The dotted lines show the decrease of the uniform field. Solid lines correspond to analytical calculations, individual points marked with squares correspond to the results of numerical calculations. The bottom row of figures shows dependences for different components of the magnetic field intensity, also normalized to the amplitude values of the corresponding field components at the surface of the conductive medium $H_{k}^{*}=\left|\dot{H}_{i k}\right| /\left|\dot{H}_{i k}(z=0)\right|$, where $k=x, y, z$. Note, in contrast to the electric field, the vertical component of the magnetic field intensity in conducting half-space in this case of a three-dimensional field is not equal to zero. (a)

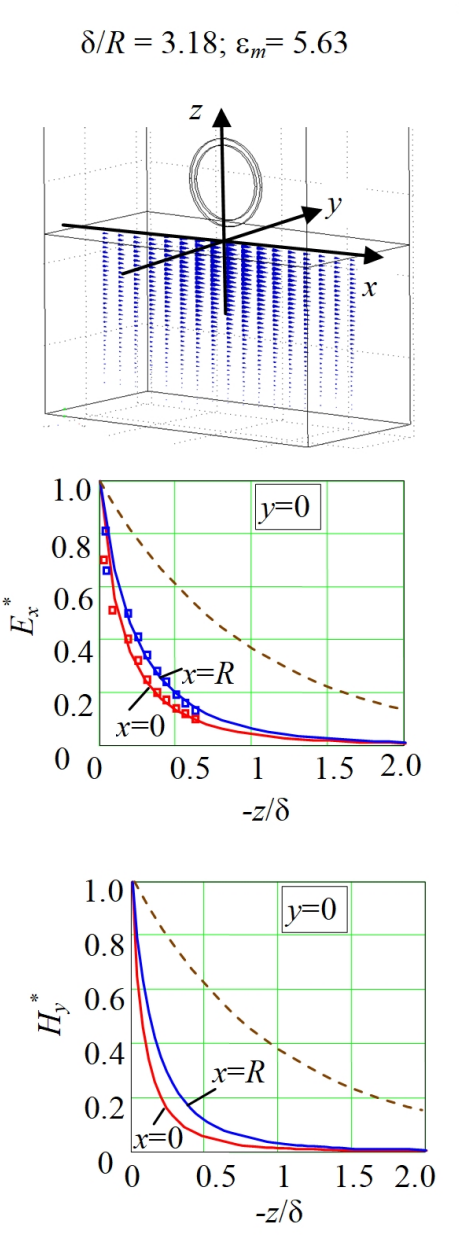

(b)
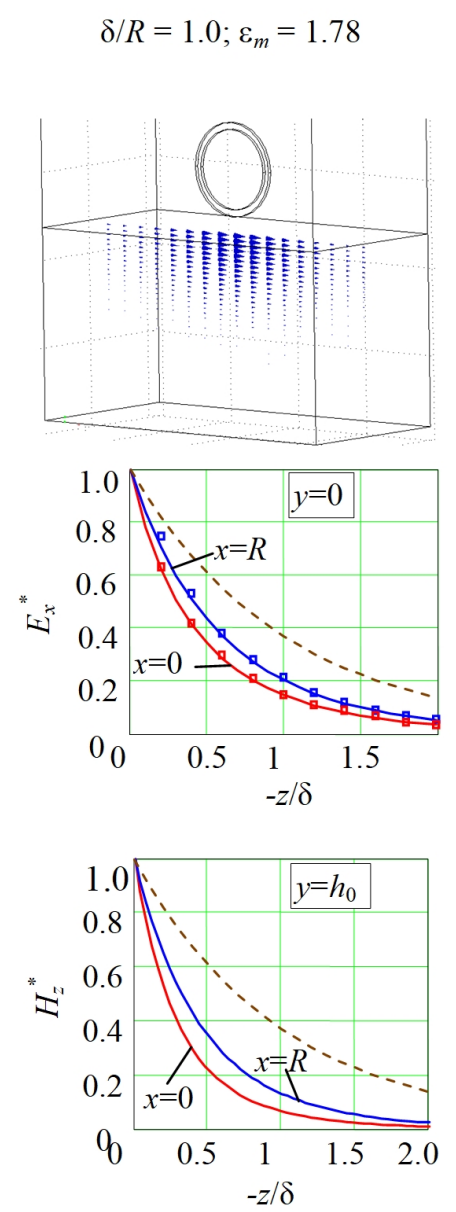

(c)
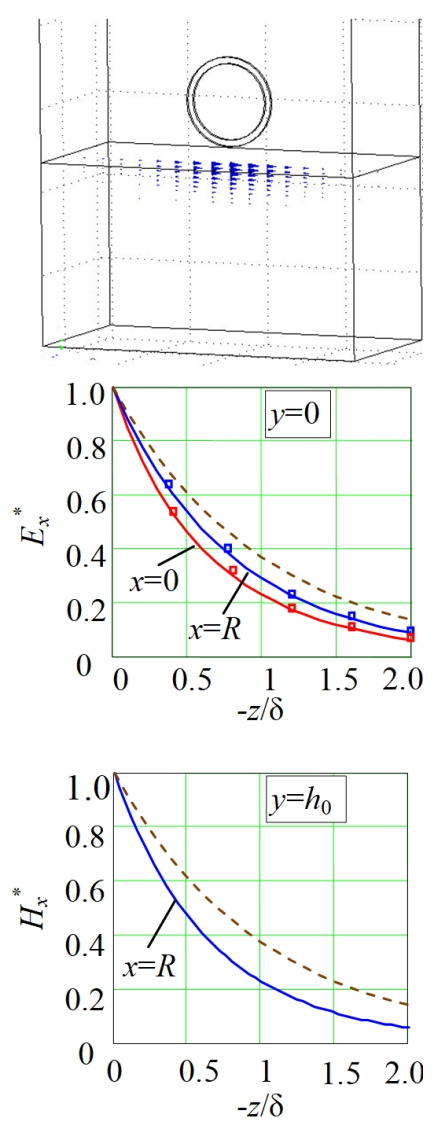

Fig. 5. Depth distribution of the normalized amplitudes for the components of the electric $E_{x}^{*}$ and magnetic $H_{x}^{*}, H_{y}^{*}, H_{z}^{*}$ intensities in conducting half-space for non-uniform three-dimensional field created by the specific system in the form of circular current contour near the flat interface between media

It is seen that with a decrease in the penetration depth $\delta$ in comparison with the radius of the contour $R$ or with the distance $h_{0}$, the penetration law both electric and magnetic fields approaches the slowest decrease of uniform field. Immediately below the contour at $x=0, y=0$, where the contour section most closely approaches to the interface, decrease is more pronounced than at $x=R$. This is explained by the fact that at $x=R$, the contour sections are at greater distance from the surface, and therefore the non-uniformity of the external field distribution near the surface is less than in the case when $x=0$.
Dashed curve in Fig. 5,b for vertical component of magnetic field has a conditional meaning, since in the approximate model of the diffusion of uniform field the component of the magnetic field intensity normal to the surface is equal to zero. However, for diffusion of a threedimensional non-uniform field, this component is nonzero.

For the considered system on the plane $x=0$, the field component $\dot{H}_{x}$ is equal to zero, and therefore in Fig. 5,c the corresponding curve is missing. 
Penetration of non-uniform electromagnetic field in the case of strong skin effect. The general feature of faster decay of non-uniform field in comparison with uniform one is the basis for analyzing the decrease of the field, when introduced parameter is small $\varepsilon<1$ and not necessarily going to zero. This parameter depends on the position of the source point $M$ on the contour. This section deals with arbitrary electromagnetic systems for which the maximum value $\varepsilon_{m}=\max (\varepsilon)$ of all $\varepsilon$ is a small parameter.

Comparison of decay of non-uniform and uniform fields. To confirm that the penetration law of non-uniform field is approached the exponential decrease of uniform field, let us compare the functions $f_{1}(z / \delta, \varepsilon, \beta)$ and $f_{2}(z / \delta, \varepsilon, \beta)$ in (4), taking into account the factor $\sqrt{1+[\chi /(\mu \sqrt{i})]^{2}}$ in the exponent with the same functions $f_{10}(z / \delta, \varepsilon, \beta)$ and $f_{20}(z / \delta, \varepsilon, \beta)$, but provided that the factor is taken to be equal to one, which corresponds to decrease of uniform field. For the electric field intensity, these functions are related to the directions of the current parallel and perpendicular to the interface. For the magnetic field intensity, the corresponding functions that follow from (3) can be similarly considered.

Let us first consider changes in functions $f_{1}(0, \varepsilon, \beta)$ and $f_{2}(0, \varepsilon, \beta)$ at the surface $(z=0)$. The dependences of the modules of these functions on the value $\rho / h=\tan \beta$ at $\mu=1$ and various values of the small parameter $\varepsilon<1$ are shown in Fig. 6. (a)

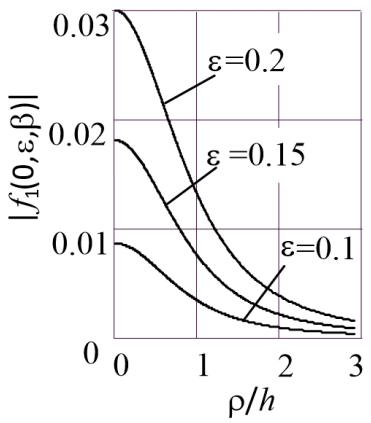

(b)

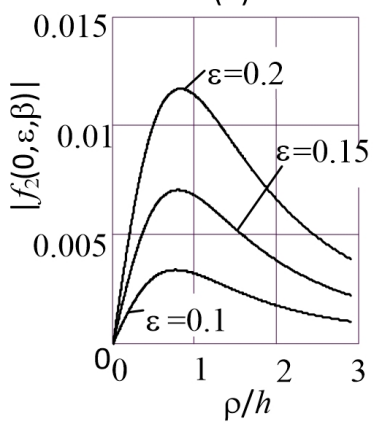

Fig. 6. Changes in the functions $\left|f_{1}(0, \varepsilon, \beta)\right|$ and $\left|f_{2}(0, \varepsilon, \beta)\right|$ at the interface depending on the relative distance $\rho / h=\tan \beta$ for small values of the parameter $\varepsilon<1$ and $\mu=1$

It can be seen that sections with different directions of the current are involved in different ways in the creation of the tangential component of the electric field intensity on the body surface. The horizontal component of the current gives the largest value of integrand in (1) just below the current element. The largest value from the vertical component of the current is realized at a certain distance from the point $M_{0}$ in the radial direction at a distance $\rho$ approximately equal to the height at which the contour element is located.

The curves in Fig. 7, $a$ show values of the modules of functions dependents on the depth for direction of the current parallel to the interface at $\mu=1$ : solid lines correspond to the function $\mid f_{1}(z / \delta, \varepsilon, \beta \mid$, dashed lines correspond to the function $\mid f_{10}(z / \delta, \varepsilon, \beta \mid$. The results are given for the case $\beta=0$ where the function $\mid f_{1}(0, \varepsilon, \beta \mid$ at the surface takes the largest values. The curves for different values of the small parameter $\varepsilon$ are obtained by choosing the corresponding values of the height $h$ above the surface on which the contour element is located. A comparison confirms the statement about the insignificant influence of the functional dependence of the integration variable in the exponential function. The quantitative values of the deviation that arise when the factor $\sqrt{1+[\chi /(\mu \sqrt{i})]^{2}}$ is replaced by one are shown in Fig. 7,b in the form of a relative deviation value $\Delta_{1}=|| f_{1}|-| f_{10}|| /\left|f_{1}\right|$. (a)

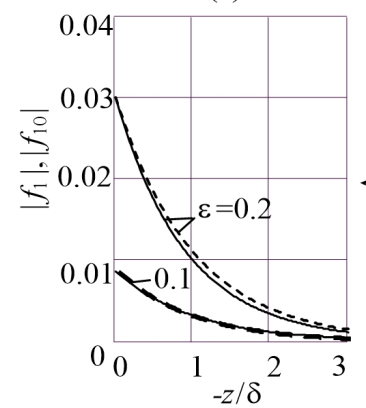

(b)

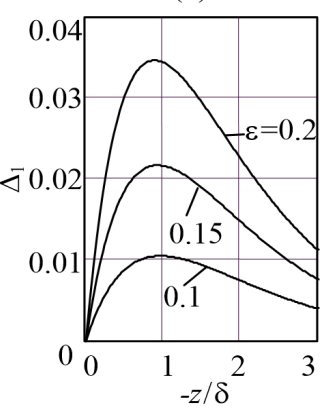

Fig. 7. Comparison of the decrease of non-uniform and uniform fields for functions $f_{1}$ and $f_{10}$ corresponding to the direction of the current parallel to the interface between media for the small value of the parameter $\varepsilon<1$ and $\mu=1$

Similar results are also valid for the term of the integrand in the contour integral (1) related to the vertical direction of the current. Comparative values of functions $f_{2}(z / \delta, \varepsilon, \beta), f_{20}(z / \delta, \varepsilon, \beta)$ and the values of their relative deviation $\Delta_{2}=|| f_{2}|-| f_{20}|| /\left|f_{2}\right|$ are shown in Fig. 8. In this case, the observation point is selected near the maximum value of the function $\left|f_{2}(0, \varepsilon, \beta)\right|$ at the interface at $\rho=h,(\beta=\pi / 4)$. (a)

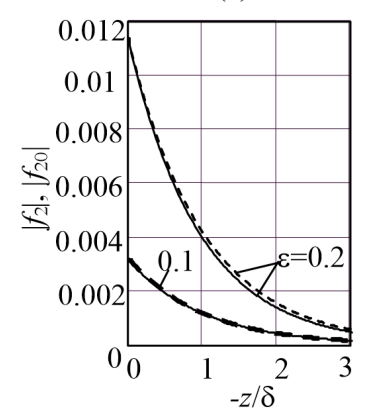

(b)

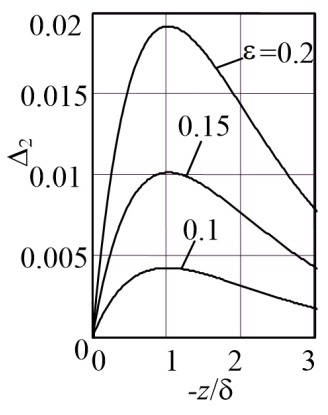

Fig. 8. Comparison of the decrease of non-uniform and uniform fields for functions $f_{2}$ and $f_{20}$ corresponding to the direction of the current perpendicular to the interface for the small value of the parameter $\varepsilon<1$ and $\mu=1$

From the presented calculations, it can be seen that with decrease in the value of the small parameter $\varepsilon$, the error from replacing the factor in the exponential by one rapidly decreases, approximately inversely proportional to the $\varepsilon^{2}$. Similar results turn out to be valid for the magnetic field intensity.

The following conclusion can be made from this. With a strong skin effect, when the maximum value of the introduced parameter $\varepsilon_{m}$ is small, the electromagnetic field decrease from the local value on the surface, approximately according to the penetration law of 
uniform field. Since the penetration law depends on the value of the parameter $\varepsilon_{m}$, the concept of strong skin effect can be extended from the point of view of the possibility of using the penetration law of uniform field. The skin effect can be considered strong when the product of the relative magnetic permeability and the penetration depth $\mu \delta$ is small not only with respect to the characteristic dimensions of conducting body, but also of the entire electromagnetic system, including the distance from the surface of body to the external sources.

Taking into account the boundary conditions $\left(z=0: \dot{\boldsymbol{E}}_{e \|}=\dot{\boldsymbol{E}}_{i \|}, \dot{\boldsymbol{H}}_{e \|}=\dot{\boldsymbol{H}}_{i \|}, \dot{\boldsymbol{H}}_{e \perp} / \mu=\dot{\boldsymbol{H}}_{i \perp}\right)$ and the expressions for the field intensities in the dielectric halfspace (7) - (9), the approximate expressions in the conducting half-space take the following form

$$
\begin{gathered}
\dot{\boldsymbol{E}}_{i} \approx e^{p z} \dot{\boldsymbol{E}}_{i}(z=0)=e^{p z} \dot{\boldsymbol{E}}_{e \|}(z=0)= \\
e^{p z} i \omega \frac{\mu_{0} \dot{I}_{0}}{4 \pi} \oint_{l} \boldsymbol{e}_{z} \times\left[\boldsymbol{t}_{1} \times\left.\nabla G_{e}\right|_{z=0}\right] d l \\
\dot{\boldsymbol{H}}_{i} \approx e^{p z} \dot{\boldsymbol{H}}_{i}(z=0)=e^{p z}\left[\dot{\boldsymbol{H}}_{e \|}(z=0)+\dot{\boldsymbol{H}}_{e \perp}(z=0) / \mu\right]
\end{gathered}
$$

where the components of the magnetic field intensities at the dielectric surface are as follows

$$
\begin{aligned}
& \dot{\boldsymbol{H}}_{e \|}(z=0)=\dot{\boldsymbol{H}}_{\boldsymbol{e} 1}(z=0)+\dot{\boldsymbol{H}}_{\boldsymbol{e} 2}(z=0)+ \\
& \left.\frac{\dot{I}_{0}}{4 \pi} \oint_{l}\left\{\left[\boldsymbol{t}_{\|} \times \boldsymbol{e}_{z}\right] \frac{\partial^{2} G_{e}}{\partial z^{2}}-\boldsymbol{t}_{\perp} \times \nabla\left(\frac{\partial G_{e}}{\partial z}\right)\right\}\right|_{z=0} d l, \\
& \dot{\boldsymbol{H}}_{e \perp}(z=0)=\frac{\dot{I}_{0}}{4 \pi} \oint_{l} \boldsymbol{t}_{\|} \times\left.\left\{\nabla\left(\frac{\partial G_{e}}{\partial z}\right)-\frac{\partial^{2} G_{e}}{\partial z^{2}} \boldsymbol{e}_{z}\right\}\right|_{z=0} d l .
\end{aligned}
$$

The expressions (10) - (12) presented as two factors are approximate only in relation to the dependence on the coordinate $z$. On the surface at $z=0$, they take into account the non-uniformity of the electromagnetic field and give the values of the field intensities without restrictions on the value of the parameter $\varepsilon=\varepsilon_{1}$.

The next two questions are related to the introduced extended concept of the strong skin effect. First, what is the difference at the interface between the intensities of the non-uniform field $\dot{\boldsymbol{E}}_{i}(z=0)$ and $\dot{\boldsymbol{H}}_{i}(z=0)$ in (10) - (12) from the values of the tangent components $\dot{\boldsymbol{E}}_{\tau}$ and $\dot{\boldsymbol{H}}_{\tau}$ for the model of the perfect skin effect. Second, what is the error of replacing the penetration law of non-uniform field with the penetration law of uniform one, depending on the value of the small parameter.

Non-uniform electromagnetic field at the interface between media. For small values $\varepsilon_{1}$, at an arbitrary point of the dielectric half-space, including at the interface between the media the expressions (10) and (11) can be simplified. In this case the function $G_{e}$ can be represented by an asymptotic series, limited by a certain number of terms $N[25]$

$$
\begin{aligned}
& G_{e} \approx \sum_{n=0}^{N} G_{n}=\sum_{n=0}^{N} \frac{2}{\sqrt{i}} a_{n}(\mu) \int_{0}^{\infty}\left(\frac{\chi}{\sqrt{i}}\right)^{n} \exp \left(-\frac{\chi \cos \beta_{1}}{\varepsilon_{1}}\right) J_{0}\left(\frac{\chi \sin \beta_{1}}{\varepsilon_{1}}\right) d \chi= \\
& =\sum_{n=0}^{N} 2(-1)^{n} a_{n}(\mu)\left(\frac{\varepsilon_{1}}{\sqrt{i}}\right)^{n+1} r_{1}^{n+1} \frac{\partial^{(n)}}{\partial z^{n}}\left(\frac{1}{r_{1}}\right)
\end{aligned}
$$

where $a_{n}(\mu)$ are the Taylor series coefficients of the function $1 / w_{1}=\sum_{n=0}^{\infty} a_{n}(\mu)(\chi / \sqrt{i})^{n}$.

The use of asymptotic expansion (13) for field intensities (1), (3) or (7), (8) on the surface made it possible to find approximate values of the field at the boundary and to establish some general features of the field formation. Finding the corresponding relations is given in [23].

Firstly, in the found analytical expressions, the electromagnetic field on the surface is determined only by the known distribution of the field of external sources at the boundary

$$
\begin{aligned}
& \dot{\boldsymbol{E}}_{\|}(z=0)=\sum_{n=0}^{N} \dot{\boldsymbol{E}}_{\| n}=\left.\varsigma \sum_{n=0}^{N} 2 a_{n}(\mu)\left(\frac{\mu}{p}\right)^{n}\left\{\frac{\partial^{(n)}}{\partial z^{n}} \boldsymbol{e}_{z} \times \dot{\boldsymbol{H}}_{0 \|}\right\}\right|_{z=0} ; \\
& \dot{\boldsymbol{H}}_{\|}(z=0)=\sum_{n=0}^{N+1} \dot{\boldsymbol{H}}_{\| n}=-\left.\sum_{n=0}^{N+1} 2 a_{n-1}(\mu)\left(\frac{\mu}{p}\right)^{n}\left\{\frac{\partial^{(n)} \dot{\boldsymbol{H}}_{0 \|}}{\partial z^{n}}\right\}\right|_{z=0} ; \\
& \dot{\boldsymbol{H}}_{i \perp}(z=0)=\sum_{n=0}^{N} \dot{\boldsymbol{H}}_{i \perp n}=-\left.\sum_{n=0}^{N} 2 \frac{a_{n}(\mu)}{\mu}\left(\frac{\mu}{p}\right)^{n+1}\left\{\frac{\partial^{(n+1)} \dot{\boldsymbol{H}}_{0 \perp}}{\partial z^{n+1}}\right\}\right|_{z=0},
\end{aligned}
$$

here, it is taken into account $\varepsilon_{1} r_{1} / \sqrt{i}=\mu / p$; it is accepted $a_{-1}=-1 ; \dot{\boldsymbol{H}}_{0}$ is the magnetic field intensity of external sources in dielectric medium at the interface. If the external field is created by a single current contour, then

$$
\dot{\boldsymbol{H}}_{0}=-\frac{\dot{I}_{0}}{4 \pi} \oint_{l} \frac{\boldsymbol{t} \times \boldsymbol{r}}{r^{3}} d l .
$$

From (14) - (16) it can be seen that the electromagnetic field is determined not only by its local value on the surface, which corresponds to $n=0$. It also depends on the derivatives of the field with respect to the coordinate, that is, on the non-uniformity of the external field at the interface between the media. In this respect, the electromagnetic field at the boundary differs from the values $\dot{\boldsymbol{E}}_{\tau}$ and $\dot{\boldsymbol{H}}_{\tau}$ in the perfect skin effect model. The difference is associated with the field of eddy currents in conducting medium, the distribution law of which depends on the degree of remoteness of the external field sources in comparison with the penetration depth. The component of the magnetic field intensity normal to the boundary, which is absent in the perfect model, is determined only by the derivatives with respect to the coordinate $z$ of the same external field component.

Secondly, the found expressions (14) - (16) made it possible to generalize the Leontovich impedance boundary condition for the case of diffusion of nonuniform electromagnetic field into conducting medium. The impedance boundary condition, which establishes the relation between the tangential components of the electric and magnetic field intensities at the interface, for individual terms of the asymptotic series is the following

$$
a_{n-1}(\mu) \dot{\boldsymbol{E}}_{\| n}=-a_{n}(\mu) \boldsymbol{s}_{z} \times \dot{\boldsymbol{H}}_{\| n} .
$$

It follows from (18) that the Leontovich approximate impedance boundary condition is valid only for the first two terms of the asymptotic series. The deviation takes 
place starting from $n=2\left(\varepsilon^{2}\right)$, for which (18) gives $\dot{\boldsymbol{E}}_{\| 2}=\left\lfloor 1-1 /\left(2 \mu^{2}\right)\right\rfloor \xi \boldsymbol{e}_{z} \times \dot{\boldsymbol{H}}_{\| 2}$. The requirement for fulfilling assumption in the approximate impedance boundary condition that the normal field component is equal to zero is more stringent. It holds only for the zero term of the asymptotic series and it is violated already at $\varepsilon^{1}$.

Influence of the small parameter value to the field penetration low with the strong skin effect. Let us perform a quantitative assessment of the influence of the small parameter value $\varepsilon$ to the change in the penetration law of the electromagnetic field into conducting half-space. The analysis is carried out based on the expansion of expressions (4) in the asymptotic series, where for small $\varepsilon$ the Taylor series expansion of the factor in the integrand is used near the zero value of the integration variable $\chi$.

Unlike (9), for the asymptotic expansion of improper integrals (4), it is necessary to use not only the expansion in a power series of the function $w_{1}^{-1}(\chi)$, but also the expansion of the exponential function (5). Taking into account, except one, next term in the expansion of the factor $\sqrt{1+[\chi /(\mu \sqrt{i})]^{2}}$, approximate expression for exponential function (5) will be as follows

$$
\exp \left(\sqrt{2 i} \frac{z}{\delta} \sqrt{1+\left(\frac{\chi}{\mu \sqrt{i}}\right)^{2}}\right) \approx\left[1+\sqrt{2 i} \frac{z}{\delta} \cdot \frac{1}{2 \mu^{2}}\left(\frac{\chi}{\sqrt{i}}\right)^{2}\right] \exp \left(\sqrt{2 i} \frac{z}{\delta}\right)
$$

where it is considered that the ratio $z / \delta$ does not exceed several units.

Taking into account (19), the functions $f_{1}(z / \delta, \varepsilon, \beta)$ and $f_{2}(z / \delta, \varepsilon, \beta)$ in (4) can be approximately represented as following (below we use the combined designation $f_{1,2}(z / \delta, \varepsilon, \beta)$ for the two functions)

$f_{1,2}\left(\frac{z}{\delta}, \varepsilon, \beta\right) \approx \exp \left(\sqrt{2 i} \frac{z}{\delta}\right)\left[f_{1,2}(0, \varepsilon, \beta)+\sqrt{2 i} \frac{z}{\delta} \cdot \frac{1}{2 \mu^{2}} k_{1,2}(0, \varepsilon, \beta)\right],(20)$

where $k_{1,2}(0, \varepsilon, \beta)$ differ from $f_{1,2}(0, \varepsilon, \beta)$ by the presence of factor $(\chi / \sqrt{i})^{2}$ in the integrands (4).

After substitution $1 / w_{1}=\sum_{n=0}^{\infty} a_{n}(\mu)(\chi / \sqrt{i})^{n}$, the functions $k_{1,2}(0, \varepsilon, \beta)$ and $f_{1,2}(0, \varepsilon, \beta)$ can be represented as expansion in asymptotic series, similarly $G_{e}$ to $(15)$ for $z=0$

$$
\left\{\begin{array}{l}
f_{1}(0, \varepsilon, \beta)=\sqrt{i} \sum_{n=0}^{N} a_{n} f_{1, n}, f_{2}(0, \varepsilon, \beta)=\sqrt{i} \sum_{n=0}^{N} a_{n} f_{2, n}, \\
k_{1}(0, \varepsilon, \beta)=\sqrt{i} \sum_{n=0}^{N} a_{n} f_{1, n+2}, k_{2}(0, \varepsilon, \beta)=\sqrt{i} \sum_{n=0}^{N} a_{n} f_{2, n+2},
\end{array}\right.
$$

where

$$
\begin{aligned}
& f_{1, n}=\int_{0}^{\infty}(\chi / \sqrt{i})^{n+1} \exp \left(-\frac{\chi \cos \beta}{\varepsilon}\right) J_{0}\left(\frac{\chi \sin \beta}{\varepsilon}\right) d \chi, \\
& f_{2, n}=\int_{0}^{\infty}(\chi / \sqrt{i})^{n+1} \exp \left(-\frac{\chi \cos \beta}{\varepsilon}\right) J_{1}\left(\frac{\chi \sin \beta}{\varepsilon}\right) d \chi .
\end{aligned}
$$

To obtain the final expressions, it is sufficient to use expressions (14) - (16) of the expansion into asymptotic series of the electric and magnetic intensities at the interface. In this case, for the additional term containing $k_{1,2}(0, \varepsilon, \beta)$, the same expressions will be valid, in which the values of the degree of functions and derivatives change from $n$ to $n+2$. Besides, since in (20) only one additional term of the series is taken into account, the functions $k_{1,2}(0, \varepsilon, \beta)$ must also contain only one term of the expansion. With the same exactness the functions $f_{1,2}(0, \varepsilon, \beta)$ can contain no more than three terms of the series. As a result, using the value of the field intensity at the interface (14), the expression in which the difference from unity for the factor in the exponential function is taken into account will be as following

$$
\dot{\boldsymbol{E}}_{i} \approx 2 e^{p z} \varsigma \boldsymbol{e}_{z} \times\left\{\begin{array}{l}
\dot{\boldsymbol{H}}_{0 \|}-\left.\frac{\mu}{p} \frac{\partial \dot{\boldsymbol{H}}_{0 \|}}{\partial z}\right|_{z=0}+\left.\left(\frac{\mu}{p}\right)^{2}\left(1-\frac{1}{2 \mu^{2}}\right) \frac{\partial^{(2)} \dot{\boldsymbol{H}}_{0 \|}}{\partial z^{2}}\right|_{z=0}+ \\
+\left.\left(\frac{\mu}{p}\right)^{2} \frac{p z}{2 \mu^{2}} \frac{\partial^{(2)} \dot{\boldsymbol{H}}_{0 \|}}{\partial z^{2}}\right|_{z=0}
\end{array}\right\}
$$

Similarly, using the values of the components of the magnetic field intensities at the boundary (15), (16) and the expansion of the exponential function (19), we can also write approximate expressions for the decrease of the non-uniform magnetic field in the conducting half-space

$$
\begin{aligned}
& \dot{\boldsymbol{H}}_{i \|} \approx 2 e^{p z}\left\{\begin{array}{l}
\dot{\boldsymbol{H}}_{0 \|}-\left.\frac{\mu}{p} \frac{\partial \dot{\boldsymbol{H}}_{0 \|}}{\partial z}\right|_{z=0}+\left.\left(\frac{\mu}{p}\right)^{2}\left(1-\frac{1}{2 \mu^{2}}\right) \frac{\partial^{(2)} \dot{\boldsymbol{H}}_{0 \|}}{\partial z^{2}}\right|_{z=0}+ \\
+\left.\left(\frac{\mu}{p}\right)^{2} \frac{p z}{2 \mu^{2}} \frac{\partial^{(2)} \dot{\boldsymbol{H}}_{0 \|}}{\partial z^{2}}\right|_{z=0}
\end{array}\right\} \\
& \dot{\boldsymbol{H}}_{i \perp} \approx-\frac{2}{p} e^{p z}\left\{\begin{array}{l}
\left.\frac{\partial \dot{\boldsymbol{H}}_{0 \perp}}{\partial z}\right|_{z=0}-\left.\frac{\mu}{p} \frac{\partial^{(2)} \dot{\boldsymbol{H}}_{0 \perp}}{\partial z^{2}}\right|_{z=0}+\left.\left(\frac{\mu}{p}\right)^{2}\left(1-\frac{1}{2 \mu^{2}}\right) \frac{\partial^{(3)} \dot{\boldsymbol{H}}_{0 \perp}}{\partial z^{3}}\right|_{z=0}+ \\
+\left.\left(\frac{\mu}{p}\right)^{2} \frac{p z}{2 \mu^{2}} \frac{\partial^{(3)} \dot{\boldsymbol{H}}_{0 \perp}}{\partial z^{3}}\right|_{z=0}
\end{array}\right\} .
\end{aligned}
$$

For the components of the electromagnetic field $\dot{\boldsymbol{E}}_{i \|}=\dot{\boldsymbol{E}}_{i}$ and $\dot{\boldsymbol{H}}_{i \|}$, directed parallel to the interface between the media, the deviation from the penetration law of uniform field takes place for the terms of series proportional to the second-order derivative of the field intensities at the surface. The deviation for the component of the magnetic field intensity perpendicular to the surface $\dot{\boldsymbol{H}}_{i \perp}$ occurs for the term of series proportional to the third-order derivative. This is due to the absence of local value of the field at the surface in (18) which already contains a common factor proportional to the value $\varepsilon$.

As follows from (23) - (25), the deviation of the penetration law of non-uniform electromagnetic field in conducting medium from the penetration law of uniform one appears when the small parameter is taken into account in the second power $\varepsilon^{2}$. This conclusion is in agreement with the calculation results shown in Fig. 7,b and $8, b$. In addition, it follows from $(23)-(25)$ that the maximum value of the modulus of the additional term takes place at the maximum value of the function $|p z \exp (p z)|=|(\sqrt{2} z / \delta) \exp (z / \delta)|$, which is realized at $-z=\delta$. This value also agrees well with the ratio $z / \delta$ in Fig. 7,b and $8, b$ when the deviation reaches its maximum value.

As can be seen from (23) - (25), for all components of the electromagnetic field with strong skin effect, the deviation of the penetration law of non-uniform 
electromagnetic field from the penetration law of uniform one is determined by the value of the same parameter

$$
\left(\frac{\mu}{p}\right)^{2} \frac{p z}{2 \mu^{2}} \sim\left(\frac{\varepsilon}{\mu}\right)^{2} \frac{z}{\delta} .
$$

Estimate (26) takes into account only the difference between the field penetration laws. The total relative error associated with the use of the model of perfect skin effect will be much more, since this model also does not take into account the field non-uniformity at the interface.

\section{Conclusions.}

The exact analytical solution of the threedimensional problem of quasi-stationary electromagnetic field in the system «current contour of arbitrary configuration - conducting half-space» allows to obtain some general substantiated consequences of the field formation. These consequences, considered in the paper, are as follows.

1. It has been established that non-uniform electromagnetic field, upon penetration into conducting half-space, decreases in depth always faster than uniform field. Quantitative characteristic of the field decrease rate can be considered the parameter proportional to the ratio of the penetration depth of uniform field to the distance from external sources to the interface between dialectic and conducting media. With decrease in this parameter, the field is decreased slower, tending to the slowest decrease of uniform electromagnetic field, when the quantitative parameter tends to zero.

2. From the point of view of the possibility of using the penetration law of uniform field the concept of a strong skin effect can be extended. The skin effect can be considered strong when the penetration depth is small not only with respect to the characteristic dimensions of conducting body, but also of the entire electromagnetic system, including the distance from the surface of body to the external sources. In this case, the introduced quantitative parameter is small.

3. In the case of strong skin effect in its extended interpretation the non-uniformity of the electromagnetic field affects both the values of the field intensities at the interface between the media and the field penetration law into conducting body. The effect of field non-uniformity at the boundary surface is expressed in the fact that the electric and magnetic field intensities, in addition to local values, contain derivatives of the external sources field with respect to the coordinate perpendicular to the interface. The found analytical expressions for the field intensities in the form of asymptotic series make it possible to generalize the Leontovich impedance boundary condition to the diffusion of non-uniform field into conducting half-space. The mathematical model of the uniform field penetration into conducting medium to study the penetration of the non-uniform electromagnetic field is valid up to the introduced small parameter in the first degree. At the same time, the use of exponential decrease of the uniform field from its value at the boundary, determined with regard for its non-uniformity, is valid up to the small parameter already in the second power. The same limitation is valid when using the Leontovich approximate impedance boundary condition.
Further theoretical work is possible in the direction of a more general description of the non-uniform field of sources, not being limited to external sources in the form of current contours. The development of the theory is also possible in the direction of the formulation of boundary value problems, taking into account the known, as it shown, field at the interface between media. The found features of the field formation can make a practical importance when simulating processes, for example, by integral methods in devices with three-dimensional electromagnetic fields. The peculiarities of the distribution of the non-uniform electromagnetic field on the surface and in the skin layer of conducting body imply the study of its influence on the energy and force characteristics, the distribution of the Poynting vector and the Maxwell stress tensor.

Acknowledgment. The research was partially supported by the project $0115 \mathrm{U} 004398$ of the Institute of Electrodynamics of the National Academy of Sciences of Ukraine.

Conflict of interest. The author declare no conflict of interest.

\section{REFERENCES}

1. Rudnev V., Loveless D., Cook R.L. Handbook of induction heating. London, Taylor \& Francis Ltd, 2017. 772 p. doi: https://doi.org/10.1201/9781315117485.

2. Lucía O., Maussion P., Dede E.J., Burdío J.M. Induction Heating Technology and Its Applications: Past Developments, Current Technology, and Future Challenges. IEEE Transactions on Industrial Electronics, May 2014, vol. 61, no. 5, pp. 25092520. doi: https://doi.org/10.1109/tie.2013.2281162.

3. Acero J., Alonso R., Burdio J.M., Barragan L.A., Puyal D. Analytical equivalent impedance for a planar circular induction heating system. IEEE Transactions on Magnetics, Jan. 2006, vol. 42, no. 1, pp. 84-86. doi: https://doi.org/10.1109/tmag.2005.854443. 4. Babutskii A.I., Chrysanthou A., Ioannou J. Effect of pulsed electric current treatment on corrosion of structural metals. Strength of Materials, 2009, vol. 41, no. 4, pp. 387-391. doi: https://doi.org/10.1007/s1 1223-009-9142-3.

5. Gallo F., Satapathy S., Ravi-Chandar K. Melting and crack growth in electrical conductors subjected to short-duration current pulses. International Journal of Fracture, 2010, vol. 167, no. 2, pp. 183-193. doi: https://doi.org/10.1007/s10704010-9543-0.

6. Vasetsky Y.M., Kondratenko I.P. Electromagnetic field of the inductor for local electric pulse effects on metal products, Technical Electrodynamics, 2020, no 4, pp. 11-14. (Ukr). doi: https://doi.org/10.15407/techned2020.04.011.

7. Psyk V., Risch D., Kinsey B.L., Tekkaya A.E., Kleiner M. Electromagnetic forming - A review. Journal of Materials Processing Technology, 2011, vol. 211, iss. 5, pp. 787-829. doi: https://doi.org/10.1016/j.jmatprotec.2010.12.012.

8. Gayakwad D., Dargar M.K., Sharma P.K., Rajesh purohit, Rana R.S. A Review on Electromagnetic Forming Process. Procedia Materials Science, 2014, vol. 6, pp. 520-527. doi: https://doi.org/10.1016/j.mspro.2014.07.066.

9. Batygin Y., Barbashova M., Sabokar O. Electromagnetic Metal Forming for Advanced Processing Technologies. Springer, Cham, 2018. doi: https://doi.org/10.1007/978-3-31974570-1.

10. Yuferev S.V., Ida N. Surface Impedance Boundary Conditions: A Comprehensive Approach. CRC Press, 2010. 412 p. doi: https://doi.org/10.1201/9781315219929. 
11. Landau L.D., Lifshitz E.M. Electrodynamics of Continuous Media. Elsevier Ltd, 1984. 475 p. doi: https://doi.org/10.1016/b978-0-08-030275-1.50024-2.

12. Knoepfel H. Pulsed High Magnetic Fields. Canada: John Wiley \& Sons, Limited, 1997. 372 p.

13. Leontovich M.A. On the Approximate Boundary Conditions for Electromagnetic Field on the Surface of Highly Conducting Bodies. Propagation of electromagnetic waves, Moscow, USSR Academy of Sciences Publ., 1948, pp. 5-20 (Rus).

14. Rytov S.M. Calculation of skin effect by perturbation method. Journal of Experimental and Theoretical Physics, 1940, vol. 10, iss. 2, pp. 180-190. (Rus).

15. Mitzner K.M. An integral equation approach to scattering from a body of finite conductivity. Radio Science, 1967, vol. 2, iss. 12, pp. 1459-1470. doi: https://doi.org/10.1002/rds19672121459.

16. Kravchenko A.N. Boundary Characteristics in Electrodynamics Problems. Kyiv, Naukova Dumka Publ., 1989. 218 p. (Rus).

17. Fridman B.E. Skin effect in massive conductors used in pulsed electrical devices: I. Electromagnetic field of massive conductors. Technical Physics, 2002, vol. 47, no. 9, pp. $1112-$ 1119. doi: https://doi.org/10.1134/1.1508074.

18. Berdnik S.L., Penkin D.Y., Katrich V.A., Penkin Y.M., Nesterenko M.V. Using the concept of surface impedance in problems of electrodynamics (75 years later). Radio physics and radio astronomy, 2014, vol. 19, no. 1, pp. 57-80. doi: https://doi.org/10.15407/rpra19.01.057.

19. Liu X., Yang F., Li M., Xu S. Generalized Boundary Conditions in Surface Electromagnetics: Fundamental Theorems and Surface Characterizations. Applied Sciences, 2019, vol. 9, no. 9, p. 1891. doi: https://doi.org/10.3390/app9091891.

20. Vasetsky Yu.M., Dziuba K.K. An analytical calculation method of quasi-stationary three-dimensional electromagnetic field created by the arbitrary current contour that located near conducting body. Technical Electrodynamics, 2017, no 5, pp. 7 17. (Rus). doi: https://doi.org/10.15407/techned2017.05.007.
21. Vasetsky Yu.M., Dziuba K.K. Three-dimensional quasistationary electromagnetic field generated by arbitrary current contour near conducting body. Technical Electrodynamics, 2018, no 1, pp. 3-12. doi: https://doi.org/10.15407/techned2018.01.003. 22. Vasetsky Yu.M., Dziuba K.K., Kucheriava I.M., Mazurenko I.L. The penetration of nonuniform electromagnetic field of current contour in conducting medium. Technical Electrodynamics, 2018, no 5, 11-14. (Ukr). doi: https://doi.org/10.15407/techned2018.05.011.

23. Vasetsky Yu. Nonuniform electromagnetic field at the interface between dielectric and conducting media. Progress in Electromagnetics Research Letters, 2020, vol. 92, pp. 101-107. doi: https://doi.org/10.2528/pierl20050802.

24. Simonyi K. Foundation of electrical engineering. Elsevier Ltd, 1963. 865 p. doi: https://doi.org/10.1016/c2013-0-02694-1.

25. Vasetsky Yu., Mazurenko I., Dziuba K. Conditions for Application of Asymptotic Method to Electromagnetic Field Analysis in System of «a Current Loop - an Eletroconducting Body». Computational Problems of Electrical Engineering, 2014, vol. 4, no. 1, pp. 91-96. Available at: http://science.lpnu.ua/sites/default/files/journalpaper/2017/sep/6032/19.pdf (accessed 20.12.2020).

Received 29.12.2020 Accepted 03.02.2020 Published 05.04.2021

Yu.M. Vasetsky ${ }^{1}$, Doctor of Technical Science, Professor,

${ }^{1}$ Institute of Electrodynamics of the National Academy of Sciences of Ukraine,

56, Peremohy Avenue, Kyiv, 03057, Ukraine,

e-mail: yuriy.vasetsky@gmail.com

How to cite this article:

Vasetsky Yu.M. Penetration of non-uniform electromagnetic field into conducting body. Electrical Engineering \& Electromechanics, 2021, no. 2, pp. 43-53. doi: 10.20998/2074-272X.2021.2.07. 